03

\title{
Столкновение одноцикловых и субцикловых аттосекундных световых импульсов В нелинейной резонансной среде
}

\author{
(C) Р.М. Архипов ${ }^{1,2}$, М.В. Архипов ${ }^{1,2}$, А.В. Пахомов ${ }^{2}$, \\ Д.О. Жигулева ${ }^{1,3}$, Н.Н. Розанов $2,4,5$ \\ ${ }^{1}$ Санкт-Петербургский государственный университет, \\ 199034 Санкт-Петербург, Россия \\ 2 Университет ИТМО, \\ 197101 Санкт-Петербург, Россия \\ ${ }^{3}$ Heinrich Heine University, \\ 40225 Düsseldorf, Germany \\ ${ }^{4}$ Государственный оптический институт им. С.И. Вавилова, \\ 199053 Санкт-Петербург, Россия \\ ${ }^{5}$ Физико-технический институт им. А.Ф. Иофрфе, \\ 194021 Санкт-Петербург, Россия \\ e-mail: arkhipovrostislav@gmail.com
}

Поступила в редакцию 12.12.2017 г.

\begin{abstract}
Теоретически на основе численного решения системы уравнений Максвелла-Блоха изучено столкновение предельно-коротких одноцикловых и униполярных субцикловых импульсов в нелинейной резонансной среде в условиях, когда взаимодействие света со средой когерентное. Рассмотрена динамика электрического поля, светоиндуцированных структур поляризации и разности заселенностей в ситуации, когда импульсы перекрываются в среде. Показано, что состояния среды (справа и слева от области перекрытия импульсов) могут различаться. В частности, продемонстрирована возможность существования волн поляризации с разными характеристиками, существующих в областях сред, расположенных по противоположным сторонам области, где импульсы перекрываются в среде. Эти волны бегут в разных направлениях и обладают разной пространственной частотой.
\end{abstract}

DOI: $10.21883 /$ OS.2018.04.45753.287-17

\section{Введение}

В настоящее время за счет метода генерации высоких оптических гармоник удается формировать предельнокороткие импульсы (ПКИ) с длительностью в аттосекундном диапазоне (1 as $=10^{-18}$ s) [1-3]. Использование таких импульсов предоставило возможность изучения многих фундаментальных вопросов, связанных со строением вещества. Например, стало возможно исследовать и управлять динамикой волновых пакетов в атомах, молекулах и твердых телах $[4,5]$, а также в металлических и диэлектрических наноструктурах [6], изучить динамику туннелирования электронов через потенциальные барьеры [7], ускорять пучки электронов [8] и т.д.

Длительность ПКИ намного меньше времен релаксации поляризации $T_{2}$ и разности заселенностей $T_{1}$ в резонансной среде. Поэтому в таких условиях, если центральная частота импульса совпадает с частотой резонанса среды, возможны проявления когерентного взаимодействия (КВ) импульса со средой. КВ проявляется в возникновении осцилляций Раби атомной поляризации и инверсии заселенностей и может приводить, например, к явлению самоиндуцированной прозрачности (СИП) [9]. При этом $2 \pi$-импульс СИП распространяется в среде без потерь. Взаимодействие ПКИ с различными средами хорошо изучено теоретически и экспериментально в условиях, когда спектр импульса находится вдали от частоты резонанса среды и проявление когерентных эффектов в виде осцилляций Раби невозможно (см. обзоры [10-13] и ссылки в них). Экспериментально изучалось когерентое распространение и эффект СИП только длинных импульсов в различных средах (газы, пары щелочных металлов) [14-16]. В последнее время удалось наблюдать эффект СИП и возникновение осцилляций Раби в полупроводниковых структурах на квантовых точках [17-20]. Следует отметить недавние эксперименты, в которых удалось наблюдать осцилляции Раби [21-25] и биения Рамзея [26] (Ramsey fringes) в квантовых точках, взаимодействующих с фемтосекундными длинными импульсами при комнатной температуре.

С другой стороны, существует немалое количество работ, в которых когерентное распространение ПКИ в резонансно-поглощающей среде изучалось теоретически [27-53]. Однако исследования, проводимые в этих работах, были в основном направлены на изучение аспектов когерентного распространения ПКИ, таких как проверка выполнимости теоремы площадей МакКолла и Хана [39-41], проверка выполнимости приближений медленно меняющихся амплитуд (ПММА) 
и вращающейся волны (ПВВ) [42-45], солитонные режимы распространения и генерация аттосекундных импульсов [27,32-38], наведение и сверхбыстрое управление светоиндуцированными структурами с помощью одноцикловых $[46,47,50]$ и субцикловых $[48,49,51]$ импульсов.

Указанные исследования проводились в основном для случая биполярных импульсов, для которых напряженность электрического поля меняет знак с течением времени и электрическая площадь импульса, определяемая как интеграл от вектора напряженности электрического поля в заданной точке пространства, равна 0 [27-47,50]. В последнее время привлекает интерес возможность получения униполярных импульсов (УИ), в которых электрическая площадь отлична от 0 [52,53]. Такие импульсы способны оказывать однонаправленное воздействие на заряженные частицы и, следовательно, эффективно передавать импульс зарядам, что позволяет использовать такие импульсы для ускорения заряженных частиц, контроля динамики волновых пакетов и т.д. [52].

Возникновение светоиндуцированных решеток разности заселенностей в случае когерентного взаимодействия с резонансной средой длинных импульсов, не перекрывающихся в среде, рассматривалось в [54-57]. Возможность наведения подобных светоиндуцированных структур поляризации и инверсии при когерентном распространении биполярных ПКИ одноцикловой длительности и УИ субцикловой длительности в резонансной среде в условиях, когда импульсы не перекрываются в среде, изучалась в [46-50]. Столкновение биполярных солитонов СИП изучалось в [5860] в случае длинных импульсов и в [42-45] в случае ПКИ. Столкновения двух униполярных субцикловых импульсов, распространяющихся в среде, состоящей из смеси усиливающих и поглощающих частиц, рассматривались в [31]. В работе [51] анализировалась динамика светоиндуцированных структур поляризации и инверсии в условиях, когда субцикловые импульсы пересекались в центре среды. Авторы [61] рассмотрели столкновительную динамику двух униполярных, субцикловых импульсов в условиях солитонного распространения импульсов в среде. В работе [66] были исследованы свойства сильно связанных долгоживущих состояний системы ,электромагнитное поле + вещество“, возникающих при столкновении когерентных импульсов в плотной резонансной среде („по-

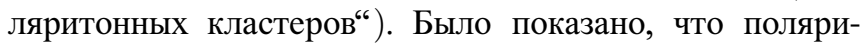
тонные кластеры обладают необходимыми свойствами базовых элементов полностью оптического сигнального процессинга.

В настоящей работе изучаются ситуации, когда в среде пересекаются и сталкиваются униполярные, субцикловые импульсы с биполярными одноцикловыми аттосекундными импульсами, а также ситуации, когда в столкновении участвует более чем 2 импульса в отличие от предыдущих исследований. Анализируется поведение светоиндуцированных структур, наводимых последовательностями таких импульсов, при разных концентрациях поглощающих частиц. Рассматривается влияние излучения бегущих волн поляризации на динамику распространяющихся импульсов. Показано, что, когда ПКИ перекрываются в среде, состояние части среды с одной стороны от области перекрытия может отличаться от состояния участка среды, находящегося с другой стороны от области, где импульсы перекрываются в среде. В частности, показано, что в областях, расположенных по разные стороны от области перекрытия импульсов, возможно образование волн поляризации с разными пространственными характеристиками. Эти волны могут распространяться в противоположных направлениях, и в случае столкновения трех импульсов в среде волны имеют разную пространственную частоту.

Отметим, что в рассматриваемом в настоящей работе случае, когда длительность импульсов сравнима с периодом световой волны, размер области перекрытия импульсов также порядка длины световой волны. Очевидно, что в этом случае невозможна интерференция падающих полей даже с кратковременным образованием стоячей волны. Поэтому размер зоны, отделяющей участки среды с разными свойствами, крайне мал. В этом существенное отличие рассматриваемого ниже случая от ситуации, когда в среде перекрываются многоцикловые биполярные фемтосекундные и более длинные импульсы. В последнем случае размер области перекрытия составляет несколько десятков, сотен и более длин волн, и создание решеток происходит за счет интерференции перекрывающихся пучков [62].

\section{Теоретическая модель и рассматриваемая система}

Для изучения столкновения униполярных субцикловых и одноцикловых импульсов аттосекундной длительности в резонансной среде применялась система уравнений Максвелла-Блоха. Ввиду малой длительности возбуждающих импульсов в ней не используются ПММА и ПВВ. Как и в работах [27-51], среда описывалась в двухуровневом приближении с использованием формализма матрицы плотности. Как показывают расчеты, основные особенности когерентного распространения в двухуровневых средах сохраняются и в случае учета большего числа уровней среды [32-34,36,50], поэтому в настоящей работе будет для простоты использовано двухуровневое приближение. Неоднородным уширением также пренебрегается. Поле считается линейно поляризованным, что сводит задачу к скалярной. Мы пренебрегаем также дифракцией излучения, что оправдано для длин распространения пучков, меньших дифракционной длины. 


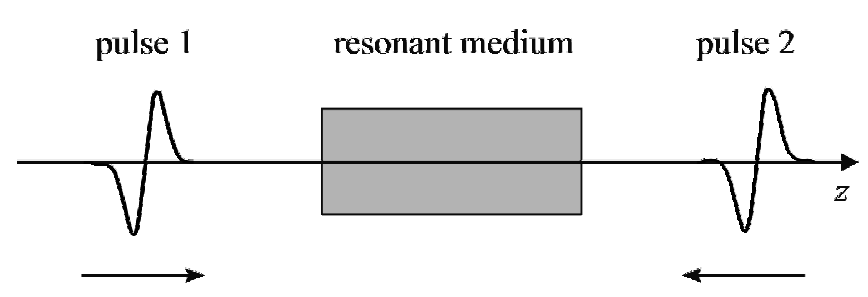

Рис. 1. Геометрия рассматриваемой системы.

Таким образом, данная система уравнений имеет вид

$$
\begin{aligned}
\frac{\partial \rho_{12}(z, t)}{\partial t}= & -\frac{\rho_{12}(z, t)}{T_{2}}+i \omega_{0} \rho_{12}(z, t) \\
& -\frac{i}{\hbar} d_{12} E(z, t) n(z, t), \\
\frac{\partial n(z, t)}{\partial t}= & -\frac{n(z, t)-n_{0}(z)}{T_{1}} \\
& +\frac{4}{\hbar} d_{12} E(z, t) \operatorname{Im} \rho_{12}(z, t), \\
P(z, t) & =2 N_{0} d_{12} \operatorname{Re}\left(\rho_{12}\right), \\
\frac{\partial^{2} E(z, t)}{\partial z^{2}}-\frac{1}{c^{2}} & \frac{\partial^{2} E(z, t)}{\partial t^{2}}=\frac{4 \pi}{c^{2}} \frac{\partial^{2} P(z, t)}{\partial t^{2}} .
\end{aligned}
$$

Системы (1)-(4) содержат следующие параметры: $P$ поляризация среды, $N_{0}$ - концентрация активных центров, $E-$ напряженность электрического поля, $c$ скорость света в вакууме, $\hbar$ - приведенная постоянная Планка, $\omega_{0}$ - частота резонансного перехода среды $\left(\lambda_{0}=2 \pi c / \omega_{0}\right.$ - длина волны резонансного перехода), $d_{12}$ - дипольный момент рабочего перехода, $n_{0}-$ разность заселенностей двух рабочих уровней при отсутствии электрического поля $\left(n_{0}=1\right.$ для поглощающей среды).

Уравнения (1), (2) описывают эволюцию недиагонального элемента матрицы плотности $\rho_{12}$ и разности $n \equiv \rho_{11}-\rho_{22}$ между диагональными элементами матрицы плотности, которая имеет смысл разности заселенности (инверсии) между основным и возбужденным состояниями двухуровневой системы. Недиагональный элемент матрицы плотности $\rho_{12}$ по формуле (3) связан с поляризацией среды, которая служит источником поля в волновом уравнении (4).

Система уравнений Максвелла-Блоха (1)-(4) активно используется в литературе для изучения когерентного распространения ПКИ в резонансной среде [27-51]. Здесь для анализа столкновительной динамики проводилось численное решение системы (1)-(4). Уравнения Блоха для матрицы плотности (1),(2) решались методом Рунге-Кутта 4-го порядка. Волновое уравнение (4) решалось методом конечных разностей.

Пространственная область интегрирования имела длину $L=12 \lambda_{0}$. Резонансная среда располагалась вдоль оси $z$ в центре области между точками $z_{1}=4 \lambda_{0}$ и $z_{2}=8 \lambda_{0}$. Распространение возбуждающих импульсов

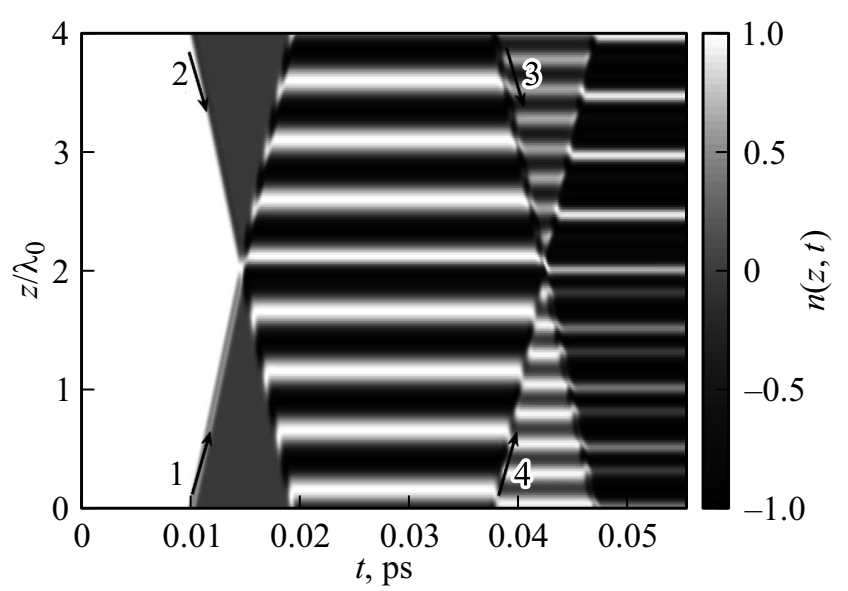

Рис. 2. Динамика разности заселенностей $n(z, t)$ под действием биполярного одноциклового импульса 1 и униполярного субциклового импульса 2 , сталкивающихся в центре среды (в точке $\left.z / \lambda_{0}=2\right)$ в момент времени $t \approx 0.015 \mathrm{ps}$. Параметры расчета указаны в таблице.

также происходило вдоль оси $z$ (рис. 1). Для создания последовательности возбуждающих импульсов в численном расчете, как и ранее в [47-51], брались нулевые граничные условия для значений поля на концах области интегрирования, что отвечает идеальному отражению излучения на границах.

\section{Столкновение биполярного одноциклового и униполярного субциклового импульса в среде}

В среду слева и справа (применительно к рис. 1) посылались ПКИ. Слева направо в среду посылался ПКИ гауссовой формы в виде (на входе в среду)

$$
E_{1}(t)=E_{01} \exp \left(-\frac{\left[t-\tau_{1}\right]^{2}}{\tau_{1 p}^{2}}\right) \sin \left(\omega_{0}\left[t-\tau_{1}\right]\right) .
$$

Справа налево - униполярный субцикловый импульс также гауссовой формы

$$
E_{2}(t)=E_{02} \exp \left(-\frac{\left[t-\tau_{2}\right]^{2}}{\tau_{2 p}^{2}}\right) .
$$

Здесь $\tau_{1,2}$ - временные задержки.

Параметры расчета приведены в таблице.

Амплитуды возбуждающих импульсов были подобраны так, чтобы импульсы выравнивали заселенности верхнего и нижнего уровней, т.е. после действия импульсов достигалась инверсия $n=0$.

Результаты расчета выявили следующую динамику системы. Рис. 2 иллюстрирует динамику инверсии $n$, а рис. 3 - динамику поляризации среды $P$. До своего столкновения импульсы переводили среду в состояние с нулевой инверсией (рис. 2) и наводили встречные (бегущие навстречу друг другу) волны поляризации (рис. 3). 


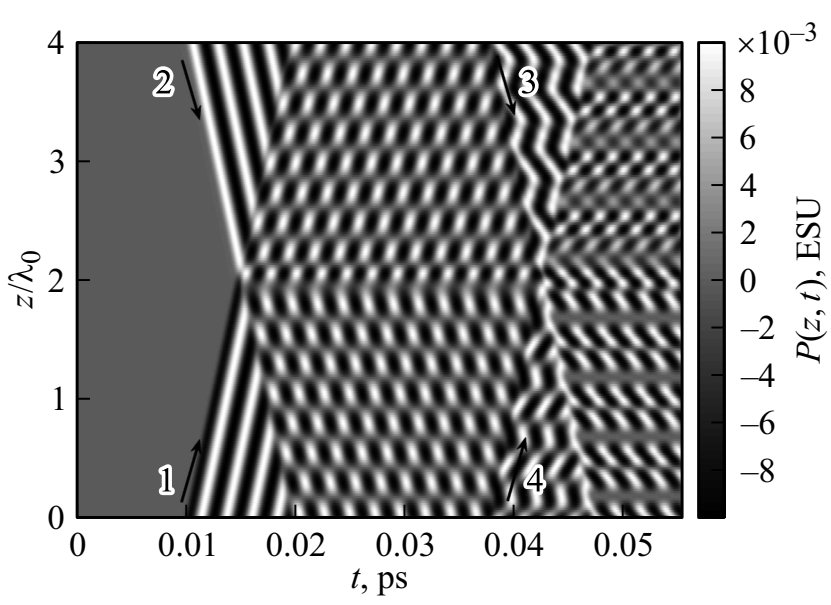

Рис. 3. Динамика поляризации $P(z, t)$ под действием биполярного одноциклового импульса 1 и униполярного субциклового импульса 2 , сталкивающихся в центре среды (в точке $z / \lambda_{0}=2$ ) в момент времени $t \approx 0.015 \mathrm{ps}$. Параметры расчета указаны в таблице.

Затем импульсы сталкивались в центре среды в точке $z / \lambda_{0}=2$ в момент времени $t \approx 0.015$ ps. Затем импульсы продолжали распространяться и начали взаимодействовать с бегущими им навстречу волнами поляризациями, наведенными ранее только что прошедшими импульсами. В результате этого в обеих половинах среды, справа и слева от точки $z / \lambda_{0}=2$, возникли решетки разности заселенностей с периодом $\lambda_{0} / 2$ (рис. 2) и волны поляризации (рис. 3). Затем импульсы выходили из среды, „отражались“ от границ области интегрирования и снова посылались в среду. Они вошли в среду около момента времени $t=0.04 \mathrm{ps}$. На рис. 2 и 3 момент входа импульсов обозначен стрелками с цифрами 3 и 4. В момент времени $t \approx 0.045 \mathrm{ps}$ происходит перекрытие импульсов в среде. До этого момента времени импульсы наводили в разных участках среды волны поляризации, бегущие в противоположных направлениях. Это отчетливо видно

Параметры, использованные при численном расчете

\begin{tabular}{l|l}
\hline \multicolumn{1}{c|}{ Дезонансного перехода } & $\lambda_{0}=700 \mathrm{~nm}$ \\
\hline Дипольный момент перехода & $d_{12}=20 \mathrm{D}$ \\
\hline Время релаксации инверсии & $T_{1}=1 \mathrm{~ns}$ \\
\hline Время релаксации поляризации & $T_{2}=1 \mathrm{ps}$ \\
\hline Концентрация атомов & $N_{0}=5 \cdot 10^{14} \mathrm{~cm}^{-3}$ \\
\hline Амплитуда поля 1 & $E_{01}=9.55 \cdot 10^{4} \mathrm{ESU}$ units \\
\hline Длительность импульса 1 & $\tau_{1 p}=388 \mathrm{as}$ \\
\hline Амплитуда поля 2 & $E_{02}=E_{01}$ \\
\hline Длительность импульса 2 & $\tau_{1 p}=\tau_{2 p}$ \\
\hline Параметр задержки & $\tau_{1}=\tau_{2}=2.5 \tau_{1 p}$
\end{tabular}

на рис. 3 в виде характерной зигзагообразной структуры слева и справа от точки $z / \lambda_{0}=2$.

Эти структуры поляризации, бегущие в противоположных направлениях в разных участках среды, пропадали после перекрытия импульсов в точке $z / \lambda_{0}=2$ в момент времени $t \approx 0.045 \mathrm{ps}$. После перекрытия импульсов в среде появились квазигармонические решетки разности заселенностей слева и справа от точки столкновения импульсов $z / \lambda_{0}=2$ и более сложные структуры волн поляризации. На рис. 4 показаны эти решетки после выхода импульсов из среды в момент времени $t=0.056 \mathrm{ps}$.

Таким образом, динамика светоиндуцированных структур при столкновении субциклового однополярного импульса и одноциклового биполярного аналогична динамике светоиндуцированных структур, возникающих при столкновении субцикловых импульсов [51] и при распространении ПКИ, не перекрывающихся в среде [46-50]. Исключением является лишь отмеченный выше интересный факт возникновения в среде участков, в которых существуют волны поляризации, бегущие в противоположных направлениях. Он не упоминался в предыдущих исследованиях [46-50].

\section{Влияние излучения волн поляризации на форму проходящих импульсов}

В предыдущем примере, как и в работах [46-51], концентрация атомов была настолько малой, что импульсы возбуждения практически не менялись при распространении. Однако наводимые бегущие волны поляризации, вообще говоря, могут излучать свет вдогонку
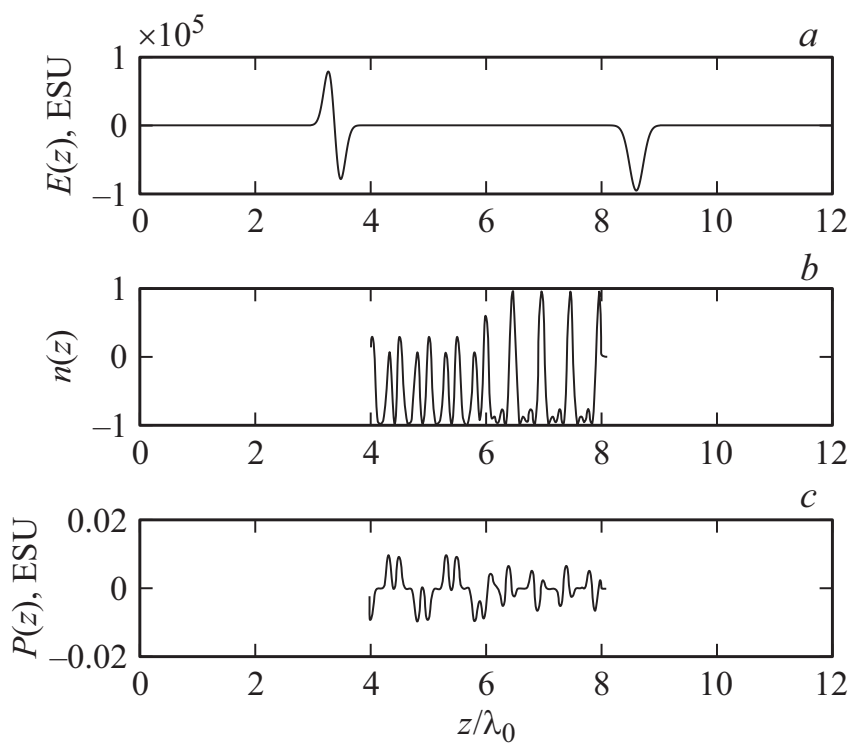

Рис. 4. $a-$ импульсы возбуждения, вышедшие из среды. Распределение разности заселенностей $(b)$ и поляризации $P(z, t)(c)$ после того, как импульсы вышли из среды для ситуации рис. 2 и 3 в момент времени $t=0.056$ ps. Среда расположена между точками $z=4 \lambda_{0}$ и $z=8 \lambda_{0}$. 

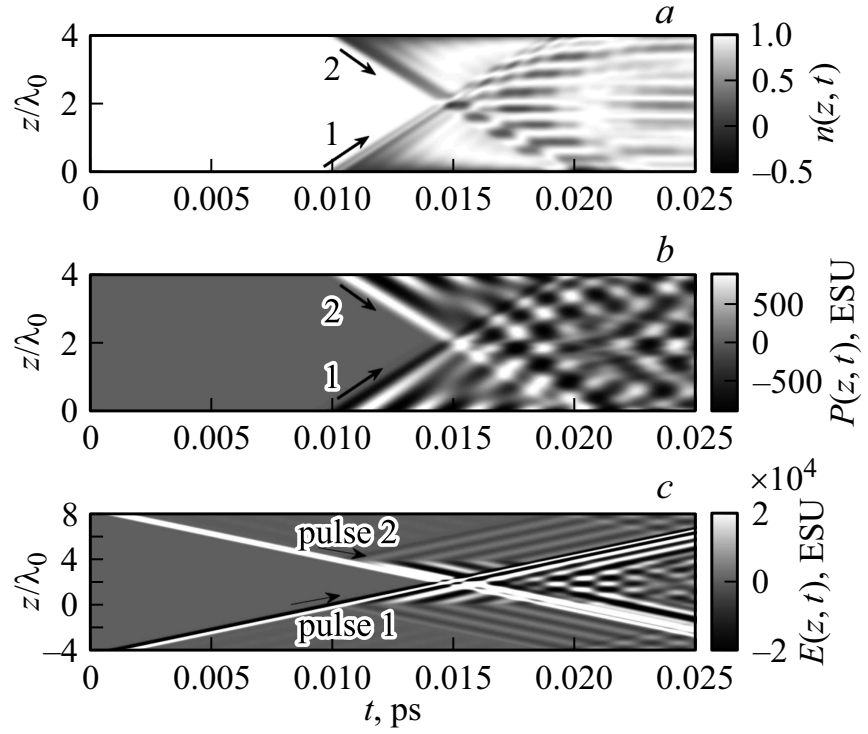

Рис. 5. Распределение разности заселенностей $(a)$, поляризации $(b)$ и поля $(c)$. Среда на $c$ расположена между точками $z=0$ и $z=4 \lambda_{0}$.

прошедшему импульсу. О такой возможности упоминалось в работе [50], однако ввиду малой концентрации поглощающих атомов величина излучаемого поля пренебрежимо мала и импульсы, распространяющиеся в среде, практически не изменяют свою форму. Интересно рассмотреть случай, когда среда оптически плотная. В последующем примере концентрация частиц имела значение $N_{0}=5 \cdot 10^{19} \mathrm{~cm}^{-3}$. Остальные параметры такие же, как в таблице.

Поведение инверсии, поляризации и поля представлены на рис. $5, a, b$ и $c$ соответственно. Импульсы входят в среду в момент времени $t=0.01 \mathrm{ps}$ и сразу начинают менять форму при распространении. Создаваемые импульсами волны поляризации (рис. 5,b) излучают свет вдогонку прошедшим импульсам, и он начинает влиять на форму прошедших импульсов. Это приводит к расплыванию импульсов по мере распространения. При этом амплитуда импульсов падает при распространении, поэтому инверсия не меняется значительно (рис. 5,a) и образование гармонических решеток не происходит. В среде наблюдается сложный обмен энергией между полем и средой. Распределение поля в пространстве, поляризации и инверсии в среде для момента времени $t=0.025$ рs показано на рис. 6 .

Интересно, что данный эффект размывания униполярных субцикловых ПКИ имеет аналогию с эффектом образования так называемых импульсов нулевой площади $(0 \pi$-импульсов) $[63,64]$. Известно, что когда импульс малой площади $\Theta$, меньшей $\pi$, и с длительностью короче времен релаксации $T_{1}$ и $T_{2}$ распространяется в резонансной среде, происходит формирование импульса нулевой площади $(0 \pi$-импульс). Этот эффект известен давно для случая длинных импульсов, когда применимо понятие площади импульса [63,64]. Если импульс с начальной площадью $\Theta<\pi$ распространяется по среде, то его площадь будет стремиться к 0 по мере распространения согласно теореме площадей Мак-Колла и Хана $[9,15,16]$. В этом случае огибающая импульса становится знакопеременной по мере распространения, и площадь импульса стремится к 0. Происходит это потому, что энергия, поглощаемая на переднем фронте импульса, переизлучается средой обратно в поле, но в противофазе. Такие $0 \pi$-импульсы были предсказаны
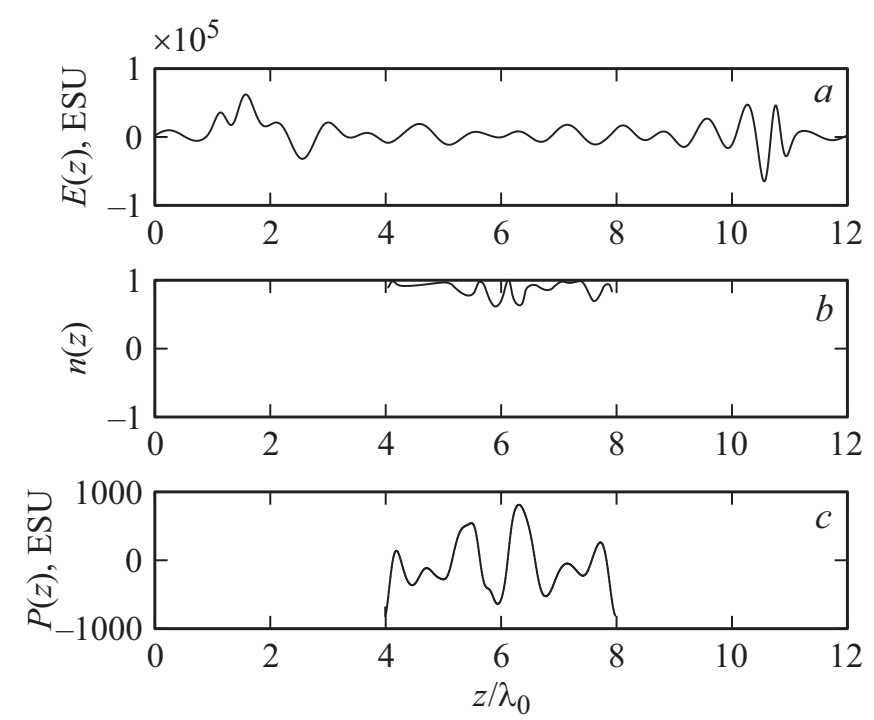

Рис. 6. Распределение поля $(a)$, разности заселенностей $(b)$ и поляризации $P(z, t)(c)$ для ситуации рис. 5 в момент времени $t=0.025 \mathrm{ps}$. Среда расположена между точками $z=4 \lambda_{0}$ и $z=8 \lambda_{0}$.

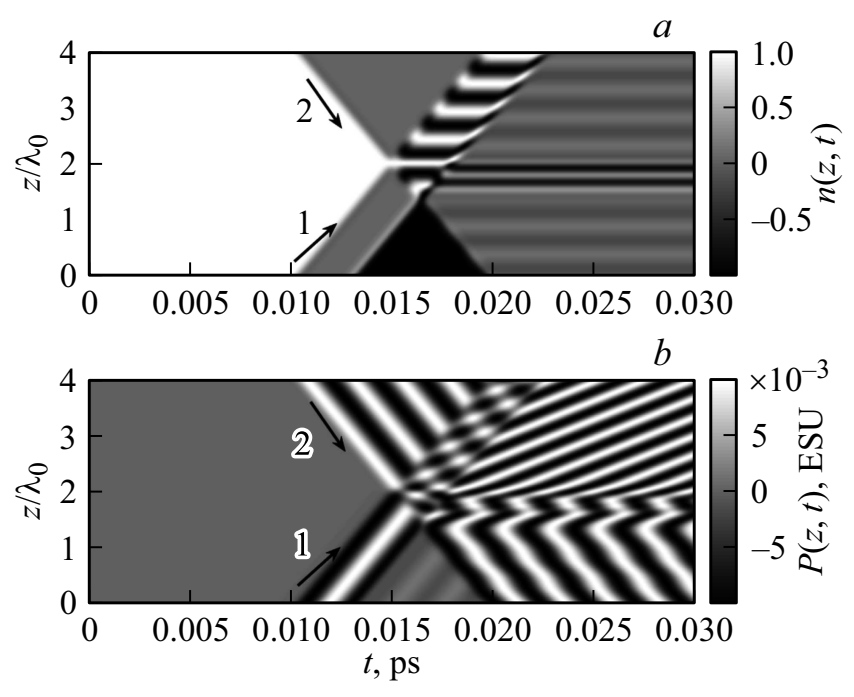

Рис. 7. Распределение разности заселенностей $n(z, t)(a)$ и поляризации $P(z, t)(b)$ при возбуждении среды полем из трех униполярных субцикловых импульсов (7) и (8). Время релаксации $T_{2}=5$ ps. Задержка $\tau_{3}=10 \tau_{1 p}$. Остальные параметры такие же, как в таблице. Стрелки показывают направление распространения импульсов. Как и в предыдущих примерах, импульсы перекрываются примерно в центре среды (в точке $\left.z / \lambda_{0}=2\right)$ в момент времени $t \approx 0.015 \mathrm{ps}$. 

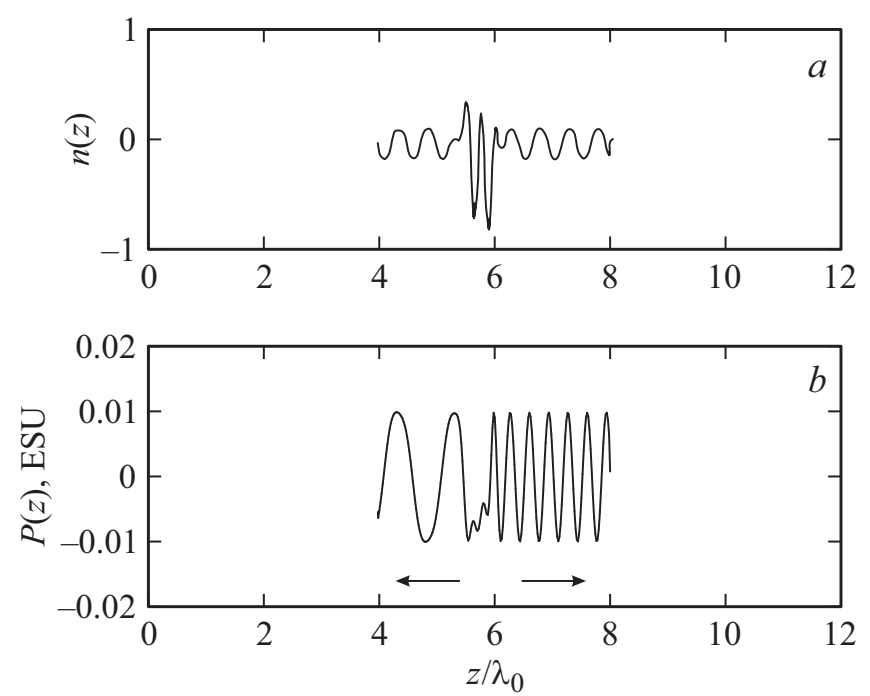

Рис. 8. Распределение разности заселенностей $n(z)(a)$ и поляризации $P(z)(b)$ в момент времени $t=0.025 \mathrm{ps}$ после того, как импульсы возбуждения покинули среду. Среда расположена между точками $z=4 \lambda_{0}$ и $z=8 \lambda_{0}$. Стрелками на $b$ показано направление распространения волн поляризации.

теоретически [63] и наблюдались экспериментально в случае сравнительно длинных импульсов [64]. Похожая динамика имеет место и в случае биполярных ПКИ одноцикловой длительности, как показано в работе [41].

\section{Столкновение трех униполярных субцикловых импульсов в резонансной среде}

В среду слева и справа посылались ПКИ (рис. 1). Поле, посылаемое слева, бралось в виде двух субцикловых импульсов с некоторой задержкой одного УИ относительно другого:

$$
E_{3}(t)=-E_{0} \exp \left(-\frac{\left[t-\tau_{1}\right]^{2}}{\tau_{1 p}^{2}}\right)-1.5 E_{0} \exp \left(-\frac{\left[t-\tau_{3}\right]^{2}}{\tau_{1 p}^{2}}\right) .
$$

Поле, посылаемое справа, также имело гауссов профиль униполярного субциклового импульса (как и ранее, имеется в виду профиль поля на левой или правой границе среды):

$$
E_{4}(t)=E_{0} \exp \left(-\frac{\left[t-\tau_{1}\right]^{2}}{\tau_{1 p}^{2}}\right) .
$$

Концентрация поглощающих атомов бралась малой, как в таблице, время релаксации $T_{2}=5 \mathrm{ps}$, амплитуда поля $E_{0}=90000 \mathrm{ESU}$ units, задержка $\tau_{3}=10 \tau_{1 p}$. Остальные параметры такие же, как в таблице.

Рис. 7 иллюстрирует динамику инверсии $(a)$ и поляризации $(b)$ в данном случае. В этом примере импульсы перекрываются в среде один раз, как и в предыдущих примерах, в центре среды (в точке $z / \lambda_{0}=2$ ) в момент времени $t \approx 0.015 \mathrm{ps}$, больше они в среду не возвращаются. Как видно из рис. 7, $a$, после перекрытия импульсов в правой половине среды образуется гармоническая решетка инверсии заселенности. Данный пример интересен тем, что после того как импульсы покинули среду, в левой и в правой половинах среды образуются гармонические волны поляризации с разной пространственной частотой (период $\lambda_{0}$ и $\lambda_{0} / 3$ соответственно (рис. 7 и $8, b$ ) после момента перекрытия импульсов $t \approx 0.015 \mathrm{ps}$ ). Эти волны поляризации, бегущие в противоположных направлениях, отчетливо видны на рис. 8, который показывает мгновенное распределение инверсии $(a)$ и поляризации $(b)$ в момент времени после того, как импульсы покинули среду. Соответственно фазовая скорость этих волн равна $c$ и $c / 3$.

Таким образом, рассмотренный пример иллюстрирует возможность создавать ситуации, когда состояние среды в левой половине отличается от состояния среды в правой половине. Как видно из предыдущего раздела, при больших концентрациях атомов существенным ста-
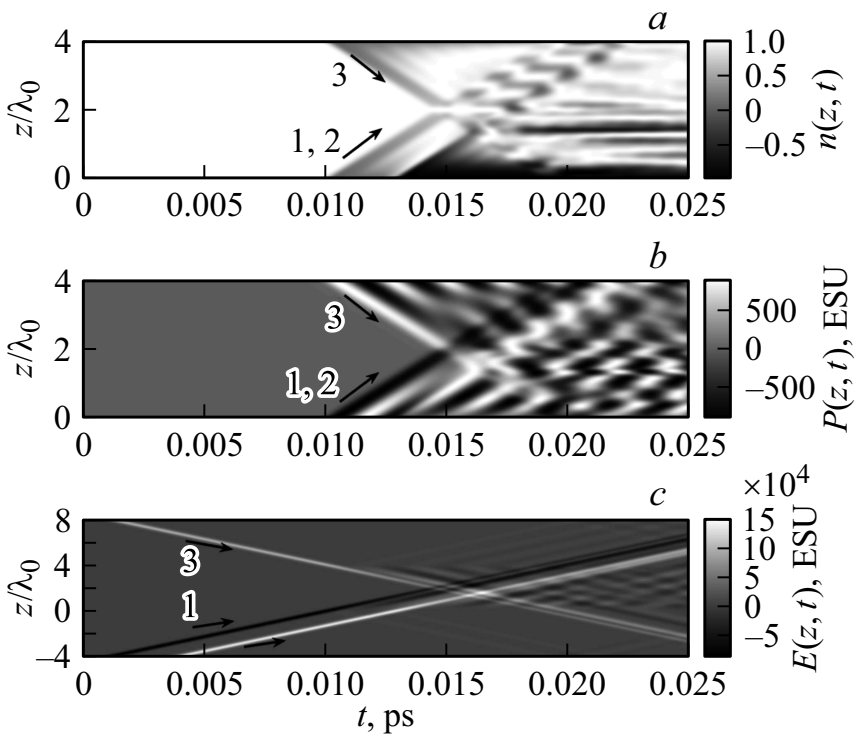

Рис. 9. То же самое, что и на рис. 6, но при большей концентрации $N_{0}=5 \cdot 10^{19} \mathrm{~cm}^{-3}$. Остальные параметры такие же, как на рис. 6. Среда на $c$ расположена между точками $z=0$ и $z=4 \lambda_{0}$.

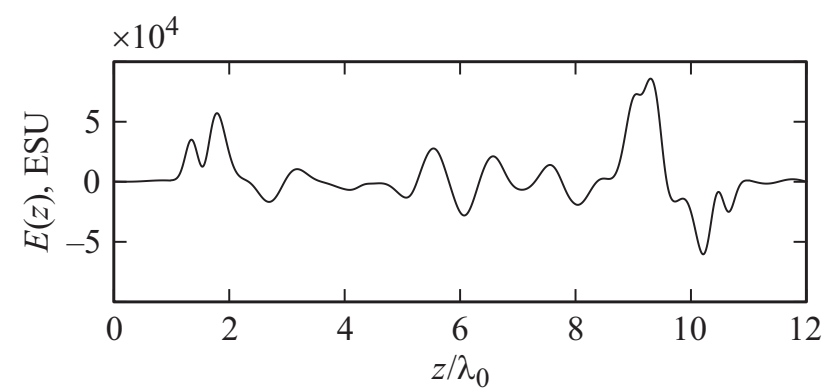

Рис. 10. Распределение поля в пространстве после того, как сталкивающиеся импульсы покинули среду в момент времени $t=0.035$ рs для ситуации рис. 8 . 
новится вклад излучения бегущих волны поляризации, что приводит к изменению формы прошедших импульсов. Соответственно следует ожидать, что при бо́льших концентрациях картина, изображенная на рис. 7, 8, будет размываться. Данное обстоятельство подтверждает и иллюстрирует рис. 9. Импульсы возбуждения также будут расплываться, что иллюстрируют рис. 9, с и 10.

\section{Заключение}

Таким образом, в работе на основе численного моделирования изучена динамика светоиндуцированных структур поляризации и инверсии в резонансной среде при распространении перекрывающихся биполярных одноцикловых и униполярных субцикловых аттосекундных импульсов, когерентно взаимодействующих со средой. Рассмотрены случаи двух перекрывающихся импульсов, когда концентрация поглощающих атомов мала и сравнительно велика. В первом случае импульсы при распространении практически не меняют своей формы, и динамика светоиндуцированных структур поляризации и инверсии заселенностей аналогична ранее исследованной авторами [46-51]. Рассмотренные волны поляризации и решетки разности заселенностей могут оставаться в среде на временах, сравнимых с временами релаксации. В случае же оптически плотной среды существенным становится тот факт, что наводимые импульсами волны поляризации являются источниками электромагнитного излучения, распространяющегося вдогонку прошедшим импульсам. Это излучение складывается с полем падающих импульсов и приводит к потере субцикловости и их расплыванию.

Наконец, был рассмотрен случай трех сталкивающихся видеоимпульсов в среде. В этом случае, как показывают расчеты, состояние среды в левой половине и правой половине может различаться. Например, в них возникают волны поляризации с троекратно различающимся пространственным периодом. Эти волны бегут в противоположных направлениях с существенно различающимися фазовыми скоростями.

Рассмотренные примеры еще раз показывают возможность сверхбыстрого управления состоянием среды на временах порядка длительности импульсов, а также хранения этих состояний на временах порядка $T_{2}$. Данные обстоятельства могут быть использованы в сверхбыстрой и нелинейной оптике для создания оптических переключателей, дефлекторов лазерного излучения и ячеек кратковременной памяти. Дифракция света на подобных структурах может быть использована для измерения времени релаксации $T_{2}$ в различных средах, что было показано ранее в случае использования длинных (наносекундных) импульсов [55,56].

Для экспериментального наблюдения подобных эффектов подходят газы или квантовые точки, обладающие бо́льшим временем релаксации поляризации $T_{2}$. Квантовые точки являются наиболее подходящей средой, поскольку они имеют дискретную структуру уровней подобно атомам, и обладают бо́льшими значениями дипольных моментов перехода (десятки Дебай). Времена релаксации поляризации $T_{2}$ в них могут при низких температурах достигать десятков и сотен наносекунд [65].

Исследование выполнено за счет гранта Российского научного фонда (проект № 17-19-01097).

\section{Список литературы}

[1] Krausz F., Ivanov M. // Rev. Mod. Phys. 2009. V. 81. P. 163.

[2] Manzoni C. et al. // Laser Photonics Rev. 2015. V. 9. P. 129.

[3] Calegari F. et al. // J. Phys. B: At. Mol. Opt. Phys. 2016. V. 49. P. 062001.

[4] Gallmann L., Cirelli C., Keller U. // Ann. Rev. Phys. Chem. 2012. V. 63. P. 447.

[5] Ramasesha K., Leone S.R., Neumark D.M. // Ann. Rev. Phys. Chem. 2016. V. 67. P. 41.

[6] Ciappina M.F., Pérez-Hernández J.A., Landsman A.S. // Rep. Prog. Phys. 2017. V. 80. P. 054401.

[7] Landsman A.S., Keller U. // Phys. Rep. 2015. V. 547. P. 1.

[8] Kozak M., McNeur J., Leedle K.J. et al. // Nature Commun. 2017. V. 8. P. 1.

[9] McCall S.L., Hahn E.L. // Phys. Rev. 1969. V. 183. P. 457.

[10] Skryabin D.V., Gorbach A.V. // Rev. Mod. Phys. 2010. V. 82. P. 1287.

[11] Dudley J.M., Genty G., Coen S. // Rev. Mod. Phys. 2006. V. 78. P. 1135.

[12] Leblond H., Mihalache D. // Phys. Rep. 2013. V. 523. P. 61.

[13] Mihalache D.U. // Romanian Reports in Physics. 2017. V. 69. P. 403.

[14] Крюков П.Г., Летохов В.С. // УФН. 1969. Т. 99. С. 169; Kryukov P.G., Letokhov V.S. // Sov. Phys. Usp. 1970. V. 12. P. 641.

[15] Полуэктов И.А., Попов Ю.М., Ройтберг В.С. // УФН. 1974. T. 114. C. 97; Poluektov I.A., Popov Yu.M., Roitberg V.S. // Sov. Phys. Usp. 1975. V. 18. P. 673.

[16] Аллен Л., Эберли Дж. Оптический резонанс и двухуровневые атомы. М.: Мир, 1978; Allen L., Eberly J.H. Optical Resonance and Two-level Atoms. NY.: Wiley, 1975.

[17] Zrenner A. et al. // Nature. 2002. V. 418. P. 612.

[18] Stievater T.H., Xiaoqin L., Stee D.G. et al. // Phys. Rev. Lett. 2001. V. 87(13). P. 133603.

[19] Borri P., Langbein W., Schneider S. et al. // Phys. Rev. B. 2002. V. 66. P. 081306.

[20] Hosseini M., Sparkes B.M., Hétet G. et al. // Nature. 2009. V. 461. (7261). P. 241.

[21] Kolarczik M. et al. // Nature Commun. 2013. V. 4. P. 1.

[22] Karni O., Capua A., Eisenstein G. et al. // Optics Express. 2013. V. 21. № 22. P. 26786.

[23] Capua A., Karni O., Eisenstein G., Reithmaier J.P. // Phys. Rev. B. 2014. V. 90. P. 045305.

[24] Karni O., Mishra A.K., Eisenstein G., Reithmaier J.P. // Phys. Rev. B. 2015. V. 91. P. 115304.

[25] Karni O., Mishra A.K., Eisenstein G., Ivanov V., Reithmaier J.P. // Optica. 2016. V. 3. P. 570-576.

[26] Khanonkin I.A., Mishra A.K., Karni O. et al. // arXiv preprint arXiv:1708.06254 (2017).

[27] Kalosha V.P., Herrmann J. // Phys. Rev. Lett. 1999. V. 83. P. 544. 
[28] Xiao J., Wang Z., Xu Z. // Phys. Rev. A. 2002. V. 65. P. 031402.

[29] Cai X., Zhao J., Wang Z., Lin Q. // J. Phys. B. 2013. V. 46. P. 175602.

[30] Lin Y., Chen I.H., Lee R.K. // Phys. Rev. A. 2011. V. 83. P. 043828.

[31] Rosanov N.N., Semenov V.E., Vyssotina N.V. // Laser Phys. 2007. V. 17. P. 1311.

[32] Высотина Н.В., Розанов Н.Н., Семенов В.Е. // Письма в ЖЭТФ. 2006. Т. 83. № 7. С. 337; Vysotina N.V., Rozanov N.N., Semenov V.E. // JETP Lett. 2006. V. 83. N 7. P. 279.

[33] Розанов Н.Н., Семенов В.Е., Высотина Н.В. // Квант. электрон. 2008. Т. 38. № 2. С. 137; Rosanov N.N., Semenov V.E., Vysotina N.V. // Quant. Electron. 2008. V. 38. N 2. P. 137.

[34] Высотина Н.В., Розанов Н.Н., Семенов В.Е. // Опт. и спектр. 2009. Т. 106. № 5. С. 793; Vysotina N.V., Rosanov N.N., Semenov V.E. // Opt. Spectrosc. 2009. V. 106. N 5. P. 713.

[35] Kozlov V.V., Rosanov N.N., Angelis C.D., Wabnitz S. // Phys. Rev. A. 2011. V. 84. P. 023818.

[36] Розанов Н.Н. Диссипативные оптические солитоны. От микро- к нано- и атто-. М.: Физматлит, 2011. Гл. 17.

[37] Song X., Yang $W$., Zeng Z., Li R., Xu Z. // Phys. Rev. A. 2010. V. 82. N 5. P. 053821.

[38] Song X., Hao Z., Yan M., Wu M., Yang W. // Laser Phys. Lett. 2015. V. 12. P. 105003.

[39] Hughes S. // Phys. Rev. Lett. 1998. V. 81 (16). P. 3363.

[40] Tarasishin A.V., Magnitskii S.A., Zheltikov A.M. // Opt. Commun. 2001. V. 193. P. 187.

[41] Tarasishin A.V., Magnitskii S.A., Shuvaev V.A., Zheltikov A.M. // Opt. Express. 2001. V. 8. P.452.

[42] Novitsky D.V. // Phys. Rev. A. 2011. V. 84. P. 013817.

[43] Novitsky D. V. // Phys. Rev. A. 2012. V. 85(4). P. 043813.

[44] Novitsky D.V. // J. Phys. B: Atomic, Molecular, and Optical Physics. 2014. V. 47 (9). P. 095401.

[45] Novitsky D.V. // Opt. Commun. 2016. V. 358. P. 202.

[46] Архипов Р.М., Архипов М.В., Бабушкин И., Розанов Н.Н. // Опт. и спектр. 2016. Т. 121. № 5. C. 810; Arkhipov R.M., Arkhipov M.V., Babushkin I., Rosanov N.N. // Opt. Spectrosc. 2016. V. 121. N 5. P. 758.

[47] Arkhipov R.M., Arkhipov M.V., Babushkin I.V., Demircan A., Morgner U., Rosanov N.N. // Opt. Lett. 2016. V. 41. P. 4983.

[48] Архипов Р.М., Архипов М.В., Пахомов А.В., Бабушкин И., Розанов Н.Н. // Квант. электрон. 2017. V. 47(7). С. 589; Arkhipov R.M., Arkhipov M.V., Babushkin I., Pakhomov A.V., Rosanov N.N. // Quant. Electron. 2017. V. 47 (7). P. 589.

[49] Arkhipov R.M., Arkhipov M.V., Pakhomov A.V., Babushkin I., Rosanov N.N. // Laser Phys. Lett. 2017. V. 14 (9). P. 1.

[50] Arkhipov R.M., Pakhomov A.V., Arkhipov M.V., Babushkin I., Demircan A., Morgner U., Rosanov N.N. // Sci. Rep. 2017. V. 7 (1). P. 12467.

[51] Архипов Р.М., Архипов М.В., Пахомов А.В., Бабушкин И., Розанов Н.Н. // Опт. и спектр. 2017. V. 123. № 4. С. 600; Arkhipov R.M., Arkhipov M.V, Pakhomov A.V., Babushkin I. // Opt. Spectrosc. 2017. V. 123. N 4. P. 610.

[52] Архипов Р.М., Пахомов А.В., Архипов М.В., Бабушкин И., Толмачев Ю.А., Розанов Н.Н. // Письма в ЖЭТФ. 2017. T. 105. № 6. C. 388. Arkhipov R.M., Pakhomov A.V., Babushkin I., Tolmachev Yu.A., Rosanov N.N. // JETP Lett. 2017. V. 105 (6). P. 408.
[53] Arkhipov R.M., Pakhomov A.V., Arkhipov M.V., Babushkin I., Tolmachev Yu.A., Rosanov N.N. // Laser Physics. 2017. V. 27. N 5. P. 053001.

[54] Abella I.D., Kurnit N.A., Hartmann S.R. // Phys. Rev. 1966. V. 141. P. 391.

[55] Штырков Е.И., Лобков В.С., Ярмухаметов Н.Г. // Письма в ЖЭТФ. 1978. Т. 27. № 12. С. 685; Shtyrkov E.I., Lobkov V.S., Yarmukhametov N.G. // JETP Lett. 1978. V. 27. N 12. P. 648.

[56] Моисеев С.А., Штырков Е.И. // Квант. электрон. 1991. T. 18. № 4. C. 447; Moiseev S.A., Shtyrkov E.I. // Sov. J. Quant. Electron. 1991. V. 21. N 4. P. 403.

[57] Штырков Е.И. // Опт. и спектр. 2013. Т. 114. № 1. С. 105; Shtyrkov E.I. // Opt. Spectrosc. 2013. V. 114. N 1. P. 96.

[58] Shaw M.J., Shore B.W. // J. Opt. Soc. Amer. B. 1990. V. 8. P. 1127.

[59] Afanas'ev A.A., Volkov V.M., Dritz V.V., Samson B.A. // J. Mod. Optics. 1990. V. 37. N 2. P. 165.

[60] Kocharovski V.V., Kocharovski Vl.V., Golubyatnikova E.R. // Computers \& Mathematics with Applications. 1997. V. 34. P. 773.

[61] Беленов Э.М., Назаркин А.В., Ущаповский В.А. // ЖЭТФ. 1991. T. 100. C. 762; Belenov E.M., Nazarkin A.V., Ushchapovskii V.A. // Sov. Phys. JETP. 1991. V. 73. P. 422.

[62] Eichler H.J., Günter E., Pohl D.W. Laser-Induced Dynamic Gratings. Berlin, Heidelberg, NY., Tokyo: Springer-Verlag, 1981.

[63] Crisp M.D. // Phys. Rev. A. 1970. V. 1. N 6. P. 1604.

64] Rothenberg J.E., Grischkowsky D., Balant A.C. // Phys. Rev. Lett. 1984. V. 53. P. 552.

[65] Bayer M., Forchel A. // Phys. Rev. B. 2002. V. 65. P. 041308.

[66] Багаев С.Н., Егоров В.С., Николаев В.Г., Чехонин И.А., Чехонин М.A. Хим. физика. 2015. Т. 34. № 8. С. 83-87; Bagaev S.N., Egorov V.S., Nikolaev V.G., Chekhonin I.A., Chekhonin M.A. A. Russ. J. Phys. Chem. B. 2015. V. 9. N 4. P. 582-586. 